\title{
Gastrointestinal endoscopy in patients on anticoagulant therapy and antiplatelet agents
}

\author{
Angelo Zulloa, Cesare Hassana ${ }^{a}$, Franco Radaellib \\ Nuovo Regina Margherita Hospital, Rome; Valduce Hospital, Como, Italy
}

\begin{abstract}
Periprocedural management of antithrombotics for gastrointestinal endoscopy is a common clinical issue, given the widespread use of these drugs for primary and secondary cardiovascular prevention. For diagnostic procedures, with or without biopsy, no adjustments in antithrombotics are usually needed. For operative procedures, balancing the risk of periprocedural hemorrhage with the continuation of antithrombotics against the chance of recurrent thromboembolic events with their discontinuation may be challenging. Oral anticoagulants need to be temporarily withheld, and consideration must be given to whether a periendoscopic "bridge" therapy, typically a low-molecular-weight heparin, should be used in order to minimize the risk of thromboembolic events. Although some emerging evidence has shown that patients receiving heparin bridging appear to be at increased risk of overall and major bleeding and at similar risk of thromboembolic events compared to controls, bridging therapy is still recommended for patients on vitamin $\mathrm{K}$ antagonists who are at high thrombotic risk. Conversely, bridging therapy is usually not needed for patients taking new oral agents, which are characterized by shorter half-lives, and a rapid offset and onset of action. Management of antiplatelet therapy requires special care in patients on secondary prevention, especially those with coronary stents. This review is intended to summarize the recommendations of updated International Guidelines designed to help the decision-making process in such an intricate field.
\end{abstract}

Keywords Gastrointestinal endoscopy, antithrombotics, direct oral anticoagulants, vitamin $\mathrm{K}$ antagonists, thrombotic risk

Ann Gastroenterol 2017; 30 (1): 1-8

\section{Anticoagulant therapy}

\section{The burden}

Anticoagulants are commonly used to reduce the risk of thromboembolic events in patients with atrial fibrillation, mechanical heart valves, deep venous thrombosis, and hypercoagulable conditions [1]. These drugs are increasingly prescribed worldwide, mostly because of the rising prevalence and incidence of atrial fibrillation. About $2 \%$ of the population

${ }^{\mathrm{a}}$ Gastroenterology and Digestive Endoscopy, Nuovo Regina Margherita Hospital, Rome (Angelo Zullo, Cesare Hassan); ${ }^{\mathrm{b}}$ Gastroenterology and Digestive Endoscopy, Valduce Hospital, Como (Franco Radaelli), Italy

\section{Conflict of Interest: Nill}

Correspondence to: Cesare Hassan, MD, Gastroenterologia ed Endoscopia Digestiva, Ospedale Nuovo Regina Margherita, Via Emilio Morosini 30, 00153 Rome, Italy, Tel.: +39 06 58446608, Fax: +39 06 58446533, e-mail: cesareh@hotmail.com

Received 28 June 2016; accepted 10 September 2016; published online 7 October 2016

DOI: https://doi.org/10.20524/aog.2016.0096 in developed countries receives long-term anticoagulation, reaching $8-10 \%$ in elderly patients [2]. After decades during which vitamin $\mathrm{K}$ antagonists (VKAs) were the only oral anticoagulation option, the spectrum of oral anticoagulants has been recently broadened by the introduction of new oral anticoagulants, also named direct oral anticoagulants (DOACs), as they directly inhibit either thrombin (dabigatran, Pradaxa $^{\oplus}$, Boehringer Ingelheim, Ingelheim, Germany) or the activated coagulation factor X (rivaroxaban, Xarelto ${ }^{\oplus}$, Bayer AG, Leverkusen, Germany; apixaban, Eliquis ${ }^{\oplus}$, Bristol-Myers Squibb, New York, NY, USA; edoxaban, Lixiana ${ }^{\oplus}$, DaiichiSankyo, Tokyo, Japan). DOACs offer several benefits over VKAs. They are prescribed at fixed doses and their anticoagulant effect is more predictable than that of warfarin, obviating the need for routine coagulation monitoring. Moreover, the rapid onset of anticoagulation (within 1-4 h) and the short half-life of DOACs (9-17 h) make initiation and interruption of therapy considerably easier than with VKAs. The individual DOACs have different pharmacologic properties, and the knowledge of these differences is important in clinical practice (Table 1).

Management of anticoagulants is a common clinical problem for gastroenterologists. Annually, $10 \%$ of patients taking anticoagulants require temporary discontinuation of therapy for surgical or other invasive elective procedures, in 
Table 1 Pharmacodynamic characteristics of novel oral anticoagulants

\begin{tabular}{lcccc}
\hline Characteristics & Dabigatran & Rivaroxaban & Apixaban & Edoxaban \\
\hline Mechanism of action & $\begin{array}{c}\text { Direct thrombin } \\
\text { inhibitor }\end{array}$ & $\begin{array}{c}\text { Direct factor Xa } \\
\text { inhibitor }\end{array}$ & $\begin{array}{c}\text { Direct factor Xa } \\
\text { inhibitor }\end{array}$ & $\begin{array}{c}\text { Direct factor Xa } \\
\text { inhibitor }\end{array}$ \\
Renal excretion & $80 \%$ & $33 \%$ & $25 \%$ & $50 \%$ \\
Time to maximum effect & $1-3 \mathrm{~h}$ & $2-4 \mathrm{~h}$ & $1-3 \mathrm{~h}$ & $1-2 \mathrm{~h}$ \\
$\begin{array}{l}\text { Elimination half-life } \\
\text { (normal renal function) }\end{array}$ & $12-14 \mathrm{~h}$ & $9-13 \mathrm{~h}$ & $8-15 \mathrm{~h}$ & $6-11 \mathrm{~h}$ \\
\hline
\end{tabular}

many cases represented by gastrointestinal (GI) endoscopy [3]. Furthermore, major GI bleeding represents a serious complication of anticoagulant therapy, with an occurrence ranging approximately from $1-4 \%$ per year and a case fatality rate up to $10 \%[4,5]$. Among patients presenting with acute upper and lower GI bleeding, up to $15 \%$ and $32 \%$, respectively take anticoagulants [6,7].

The periendoscopic management of patients receiving anticoagulants, for either urgent or elective procedures, is challenging, as the thrombotic and bleeding risks may vary depending on individual circumstances. Practice guidelines from the European Society of Gastrointestinal Endoscopy (ESGE) [8-10] and the American Society of Gastrointestinal Endoscopy (ASGE) [11], recently updated, may assist the physician in the decision-making process.

\section{Anticoagulants and urgent endoscopy}

Both the ESGE and the ASGE guidelines strongly recommend temporary interruption of anticoagulant therapy in all patients who present with clinically significant acute GI bleeding $[9,10]$. In the setting of VKA-based therapy, the decision to reverse anticoagulation should be based on the clinical assessment of the severity of bleeding at presentation. Evidence to support different strategies for the correction of coagulopathy is lacking, and this may potentially explain the large variation in practice among endoscopists [12]. The updated ESGE guidelines on upper GI bleeding have endorsed the 2008 recommendation by the American College of Chest Physicians (ACCP) [5] to administer prothrombin complex concentrate (PCC) or fresh frozen plasma, if PCC is unavailable, in addition to intravenous vitamin $\mathrm{K}$ in patients with serious, life-threatening bleeding (i.e. patients presenting with persistent or intermittent hemodynamic instability), in the case of either therapeutic or supratherapeutic elevations of the international normalized ratio (INR). For patients who are not actively bleeding and are hemodynamically stable, intravenous vitamin $\mathrm{K}$ administration may be an option.

Early endoscopy is encouraged, especially if an upper GI bleeding source is likely, and correction of coagulopathy should not delay it, according to data from two observational studies suggesting that endoscopic therapy is safe and effective even in patients with moderately elevated $\operatorname{INR}(\leq 2.5)[13,14]$. Conversely, there are no data concerning the safety and effectiveness of endoscopic therapy in bleeding patients whose supratherapeutic INR has not been corrected. Therefore, partial or complete reversal of coagulopathy before endoscopy is strongly advised in cases of serious or life-threatening bleeding. In patients at high thrombotic risk with no severe bleeding, delaying endoscopy may be a reasonable option, taking into account that the use of reversal agents carries a small but not negligible risk of thromboembolic complications and thus needs judicious use in these patients.

Acute GI bleeding in the setting of DOACs, in contrast to warfarin, requires a different approach, because of the drugs' distinct pharmacology. Given the relatively short half-lives of DOACs, their clearance and the consequent loss of anticoagulation after drug interruption are rapid and predictable, occurring gradually over $12-24 \mathrm{~h}$ in the absence of renal or hepatic failure. Thus, time is the most important antidote for DOACs, and the use of reversal agents is less important than in the case of warfarin. If the patient is hemodynamically stable and/or responds to resuscitation, it may be reasonable to simply observe the patient closely and defer endoscopy for 12-24 $\mathrm{h}$, thus allowing the natural clearance of the drug and the resumption of normal hemostatic functions. Supportive measures with aggressive fluid replacement are important for enhancing renal excretion, which may deteriorate during an acute bleeding event. Conversely, if the patient is persistently or intermittently unstable, emergent endoscopy may be appropriate, and strategies to accelerate anticoagulation reversal should be considered whenever clinically significant DOAC levels are suspected [10,15]. In this regard, it is important to underline that routine laboratory tests are not sensitive for the quantitative assessment of the anticoagulant intensity of DOACs, although a normal prothrombin time and activated partial thromboplastin time usually exclude an anticoagulation effect due to rivaroxaban and dabigatran, respectively. Apixaban has little or no effect on these tests, which may be normal in the presence of clinically relevant drug concentrations [16]. In the absence of more appropriate laboratory assays to determine the anticoagulant activity (diluted thrombin time for dabigatran or anti-Xa specific calibrators, not yet available in most centers), the presence of a clinically relevant DOAC concentration can be roughly estimated by taking into account the timing of the last dose intake, the drug's half-life, and creatinine clearance $(\mathrm{CrCl})$.

Currently, a specific antidote is available only for dabigatran (idarucizumab), although specific antidotes for the other drugs (andexanet alpha, aripazine) are expected to be available soon. Idarucizumab (Praxbind ${ }^{\circledast}$, Boehringer Ingelheim) is a monoclonal antibody fragment that binds dabigatran, 
neutralizes its activity with an affinity that is 350 -fold higher than thrombin, and it is able to completely reverse its anticoagulant effect within minutes [17]. This drug is now approved in both US and Europe for use in case of life-threatening, uncontrolled bleeding or prior to emergency surgery.

The use of non-specific pro-hemostatic agents, including activated and non-activated PCCs and recombinant factor VIIa, may be considered for life-threatening bleedings associated with direct factor Xa inhibitors until specific antidotes become available. However, supportive clinical data are limited to healthy human volunteers, animal models and in vitro studies, and a few anecdotal accounts [15]. No study has assessed their clinical efficacy and safety in patients with active bleeding.

As regards the resumption of anticoagulants following interruption, both European and US guidelines recommend restarting therapy in all patients who have an indication for long-term anticoagulation. According to a recent metaanalysis, the resumption of VKAs is associated with a significant reduction in thromboembolic events (hazard ratio [HR] 0.68, 95\% confidence interval [CI] 0.52-0.88) and mortality (HR $0.76,95 \%$ CI $0.66-0.88)$, and with a non-significant increase in rebleeding (HR 1.20, 95\% CI 0.66-0.88) [18]. The timing of anticoagulant resumption should be assessed on a patient by patient basis. In a large observational study, restarting warfarin therapy within 7 days from the index bleeding event was associated with an approximately twofold increased risk of rebleeding. Conversely, as compared with resuming warfarin beyond 30 days, resumption within between 7 and 30 days did not increase the risk of rebleeding, but did significantly decrease the risk of thromboembolism while improving survival [19]. These data support the ESGE recommendations that resumption of anticoagulation between 7 and 15 days following the bleeding event is safe and effective in preventing thromboembolic complications for most patients. Earlier resumption, within the first week, may be indicated for patients at high thrombotic risk (e.g. chronic atrial fibrillation with previous embolic event, $\mathrm{CHADS}_{2}$ score $\geq 3$, mechanical prosthetic heart valve, recent deep venous thrombosis or pulmonary embolism, known severe hypercoagulable state). In these selected cases, bridging therapy with heparin may also be considered [15]. No data are currently available to guide the timing of DOAC resumption following a bleeding event. It can be hypothesized that the principles adopted for VKAs (i.e. resumption of anticoagulation between 7 and 15 days following the bleeding event) could be extended to DOACs; however, caution is required because of their rapid onset of action.

\section{Anticoagulants and elective endoscopy}

The recommendations for anticoagulant management are anchored to the key principle of patient stratification into risk categories according to procedure-related bleeding and the underlying indication for long-term anticoagulation, as shown in Fig. 1. In this regard, there are some differences between the European [8,9] and the US guidelines [11], which deserve to be outlined. Traditionally, low-risk procedures include diagnostic endoscopy, with or without mucosal biopsies, and biliary or pancreatic stenting without sphincterotomy. The ASGE guidelines also include in this category some operative procedures with rates of bleeding of $1.5 \%$ or less among patients not receiving antithrombotic agents, such as argon plasma coagulation, Barrett's ablation, and enteral stent deployment. As concerns the thrombotic risk, the ESGE guidelines dichotomize patients into low- or high-risk, while the ASGE guidelines favor the classification of patients into three risk classes (high, medium and low), as proposed by the ACCP [3]. This simplification appears to be very practical, as it clearly identifies patients on VKAs requiring (high-risk) or not requiring (low-risk) "bridging anticoagulation", i.e., therapeutic doses of heparin (typically low-molecular weight heparin [LMWH]) to minimize the risk of perioperative thromboembolism during the period while oral anticoagulation is suspended. On the other hand, the ESGE recommendations for heparin bridging might be criticized, as they exclude patients with conditions traditionally considered to entail a high risk of thromboembolic events, such as those with non-valvular atrial fibrillation and a prior thromboembolic event, and/or a CHADS 2 score of 5 or 6 , and those with recent (within 3 months) venous thromboembolism [3]. A recent trial involving 1884 patients with atrial fibrillation, who underwent perioperative interruption of warfarin and were randomized to bridging with LWMH or placebo, demonstrated that forgoing bridging anticoagulation was non-inferior to perioperative bridging for the prevention of arterial thromboembolism and decreased the risk of major bleeding [20]. However, the actual evidence is probably not sufficient to alter the ACCP recommendation for the use of heparin in patients at higher risk for thromboembolism. Indeed, the proportion of patients with a $\mathrm{CHADS}_{2}$ score of 5 or 6 was low $(\leq 3.4 \%)$, and the study was not designed for this subgroup analysis. Results of a randomized controlled trial comparing bridging versus no bridging in patients at high risk of thromboembolism (PERIOP-2) are eagerly awaited [21].

\section{Warfarin}

Practice guidelines summarize management strategies by identifying three typical case scenarios: 1) patients undergoing low-risk endoscopic procedures; 2) patients at low thrombotic risk undergoing high-risk procedures; and 3) patients at high thrombotic risk undergoing high-risk procedures. The general principle is that anticoagulants can be continued for low-risk procedures and must be suspended for all high-risk ones. For low-risk procedures, the INR should be checked during the week before the procedure to ensure that it does not exceed the therapeutic level. However, routine discontinuation of warfarin may be considered even for diagnostic procedures that have a relevant probability of becoming high-risk, an example being the case of polypectomy during colonoscopy. In this specific regard, recent data support the safety of colonic polypectomy of small $(\leq 10 \mathrm{~mm})$ polyps without interrupting warfarin, provided that endoscopic clips are routinely applied prophylactically [22] or a cold snare is used, rather than conventional polypectomy, in order to reduce the risk of delayed bleeding [23]. However, further data are necessary before current practice can be changed. 


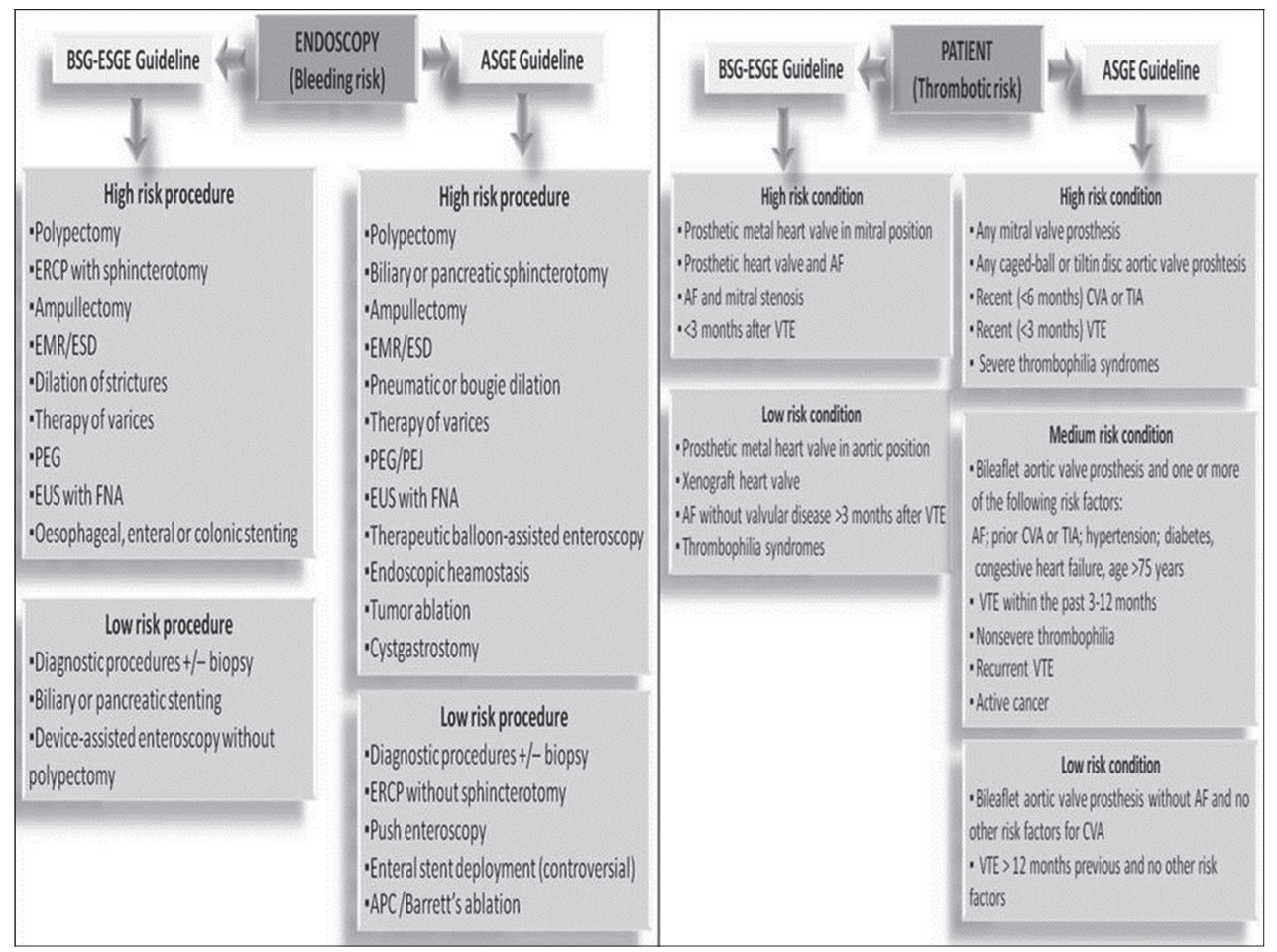

Figure 1 Bleeding risk stratification according to endoscopic procedure in the European and US guidelines ERCP, endoscopic retrograde cholangiopancreatography; EMR/ESD, endoscopic mucosal resection/endoscopic submucosal dissection; $P E G$, percutaneous endoscopic gastrostomy; EUS, endoscopic ultrasound; FNA, fine-needle aspiration; PEJ, percutaneous endoscopic jejunostomy; APC, Argon plasma coagulation; AF, atrial fibrillation; VTE, venous thromboembolism; CVA, cerebrovascular accident; TIA, transient ischemic attack

For high-risk procedures in patients at low thrombotic risk, it is recommended to discontinue warfarin 5 days before the examination. The INR decreases to $\leq 1.5$ in approximately $93 \%$ of patients within 5 days after interruption. Longer intervals may be needed if the INR remains in the therapeutic range.

Shorter intervals (last dose taken 3 days preoperatively) are appropriated for patients who are taking other coumarin anticoagulants with shorter half-lives than warfarin, such as acenocoumarol. The INR should be checked within $24 \mathrm{~h}$ before the procedure, to ensure that its value is below 1.5. Post-procedure, warfarin can be restarted at the usual daily dose on the same day, because the achievement of full anticoagulation after the reinstitution of warfarin takes several days.

For high-risk procedures in patients who are at high thrombotic risk, it is recommended to discontinue warfarin 5 days before the procedure, to start a daily therapeutic dose of LWMH (100 UI/kg b.i.d.) 3 days before the examination, and to withhold it $24 \mathrm{~h}$ prior to the procedure. Subsequently, warfarin can be resumed at the usual daily dose on the day of the procedure, while the daily dose of LWMH should be restarted on the following day, if the risk of post-procedural bleeding is deemed acceptably low, and continued until a therapeutic INR is achieved (approximately 5 days later). In patients believed to be at high risk for adverse bleeding events, delayed restarting of LWMH $48 \mathrm{~h}$ after the procedures can be a reasonable option, according to the 2012 ACCP guidelines [3]. LMWH are usually preferred to unfractionated heparin, as they have similar efficacy, even in high-risk patients such as those with metal heart valves, but are more convenient to use and do not require intensive monitoring [24]. Intravenous unfractionated heparin may be a reasonable alternative in patients with renal insufficiency and/or those requiring hemodialysis, as their dosing is unaffected by renal clearance.

Some GI endoscopic procedures are associated with cauteryinduced injury, which may result in delayed bleeding, usually 7-10 days after the procedure. The resumption of antithrombotic therapy, particularly full-dose therapy, is a major determinant of the bleeding risk after invasive procedures, and the use of bridging anticoagulation further increases this risk. Thus, all patients should be advised that there is an increased risk of post-procedural bleeding compared to non-anticoagulated patients $[8,9]$.

\section{DOACs}

Because of their short half-life, DOACs can generally be continued until shortly before the procedure, and because of their rapid onset of action, therapeutic anticoagulation is achieved within a few hours of reinstituting treatment. These pharmacodynamic properties obviate the cost and inconvenience of "bridging anticoagulation" with heparin. In this regard, it is also worth noting that patients with the highest thrombotic risk (i.e., those with mechanical heart valve prostheses) are excluded from the DOACs indications. 
In the case of low-risk elective GI procedures, such as endoscopy or colonoscopy with biopsy, the ASGE guidelines suggest continuing DOACs in the periendoscopic period, following the same management strategy as for VKAs [11]. Safety concerns may arise in relation to this management strategy, when we consider that in a patient taking DOACs the intensity of anticoagulation may be relatively high compared to the average intensity at the peak levels, which is reached about 2-4 $\mathrm{h}$ after oral administration. Conversely, the procedure should ideally be performed when the DOAC is at trough levels, and the anticoagulant effect is relatively low. This means that the optimal procedure window is roughly $10 \mathrm{~h}$ after the last dose intake for the drugs given twice daily (dabigatran and apixaban), and $20 \mathrm{~h}$ for rivaroxaban, which is given once daily. On the basis of these considerations, and until further safety data in this specific situation become available, the ESGE guidelines suggest omitting the morning DOAC dose on the day of a low-risk procedure, so that biopsies can be sampled at a trough level $[8,9]$.

In the case of high-risk procedures, it is mandatory to withhold DOACs. DOAC interruption 2 days before the procedure corresponds to approximately 4-5 half-lives elapsed between stopping the anticoagulant and endoscopy. This ensures a minimal (3-6\%) residual anticoagulant effect at the time of endoscopy in the majority of patients, allowing procedures with a high risk of bleeding to be performed safely. Although all DOACs have some degree of renal excretion, dabigatran's pharmacokinetics are the most influenced by renal function, as $80 \%$ of the drug is cleared by the kidney. Thus, for dabigatran an additional day of interruption (3 days overall) is required in patients whose renal function is moderately impaired $(\mathrm{CrCl} 30$ $49 \mathrm{~mL} / \mathrm{min}$ ) to ensure elimination of any residual anticoagulant effect. For other DOACS, a longer interruption is not needed, as there is no significant accumulation of the drug even in case of severe renal dysfunction $(\mathrm{CrCl}<30 \mathrm{~mL} / \mathrm{min})$. Besides, dose reduction is usually recommended in these patients.

According to these considerations, in the case of high-risk elective GI procedures, the ESGE guidelines recommend that the last dose of DOACs be taken at least $48 \mathrm{~h}$ before the procedure. That means that the last dose should be given 3 days before endoscopy. For patients on dabigatran with a $\mathrm{CrCl}$ of $30-50 \mathrm{~mL} / \mathrm{min}$, the last dose should be taken 4 days prior to the procedure $[8,9]$.

DOACs can be resumed after the procedure, provided that hemostasis has been achieved, at the same dose the patient was receiving pre-procedure. It is of utmost importance for clinicians to be aware that, unlike the reintroduction of warfarin, which results in delayed anticoagulation for several days, in the case of DOACs a therapeutic intensity of anticoagulation is restored within $3 \mathrm{~h}$ after a single dose intake. Therefore, the ESGE guidelines suggest delaying the reintroduction of DOACs for $48 \mathrm{~h}$ after a high-risk procedure. However, for procedures with a significant risk of delayed hemorrhage, such as a large endoscopic mucosal resection (EMR) or endoscopic submucosal dissection (ESD), a longer period of discontinuation may be considered $[8,9]$.

Because of the pharmacokinetic profile of DOACs, the rationale for heparin bridging in this setting is much weaker than when VKAs are interrupted [25]. Data from prospective registries clearly indicated that patients who received bridging anticoagulation had similar rates of thrombotic events compared with those who did not receive bridging, but experienced an up to 5 -fold greater risk of major bleeding [26,27]. These findings do not support the use of LMWH bridging during interruption of DOAC therapy for elective procedures.

\section{Antiplatelet therapy}

\section{The burden}

Antiplatelet agents (APAs) are very useful drugs that are widely used in clinical practice, mainly for cardiovascular prevention [28]. A recent Italian survey, involving 3433 consecutive patients visited by primary care physicians for any reason, found that as many as $25.3 \%$ of them were on chronic APA therapy [29]. Although the clinical benefits are generally superior to the risks [30], APAs may cause GI and large-bowel bleeding [31-33]. More than half the patients who present nonvariceal bleeding have been found to be on non-steroidal antiinflammatory therapy [34-37]. In addition, the probability of serious upper GI bleeding events is particularly high when two APAs were administered, the risk being increased 1.8-fold with low-dose aspirin alone and 7.4-fold with aspirin and clopidogrel dual therapy [38]. Dual APA therapy is increasingly used in clinical practice, not only in patients with drug-eluting coronary stents, but also in those at high thrombotic risk [39]. Indeed, a meta-analysis found that aspirin and clopidogrel dual therapy was more effective than aspirin alone in reducing both the risk of primary ischemic stroke and its recurrence in patients with a previous stroke/transient ischemic attack [39]. In a Japanese series of 3492 endoscopies, as many as $448(12.8 \%)$ patients were on APA therapy, while 124 of these $(27.7 \%)$ were on dual, triple, or even quadruple therapy [40]. Therefore, endoscopists are not infrequently required to perform an urgent endoscopy in a bleeding patient in ongoing APA therapy [41].

On the other hand, APAs increase the risk of bleeding during endoscopy, particularly following different operative procedures that are routinely performed in clinical practice. However, major bleeding - requiring endoscopy, transfusion, hospitalization - may occur even following a cold biopsy. Retrospective studies have suggested that the risk of overt bleeding from cold mucosal biopsy overall ranges from 0.02 $0.1 \%$, being distinctly higher in those taking APAs $[42,43]$, and it increases with the number of biopsies [43]. Indeed, previous Japanese guidelines recommended discontinuation of antithrombotic agents before cold biopsy [44], while former Australian guidelines suggested that it was reasonable to withhold aspirin 5-7 days prior to endoscopy, restarting it either immediately afterwards or 10-14 days later if a therapeutic procedure has been performed [45].

Based on all these issues, the endoscopist should interrupt APA therapy 5-7 days before endoscopy in all patients to minimize the bleeding risk, especially when considering that an unplanned operative procedure (e.g. a polypectomy) might be necessary. Such a cautious approach is surely also advantageous 
for the patient in terms of bleeding prevention. Nevertheless, following this procedure, the potential cardiovascular risk related to the APA interruption is entirely neglected [46]. If the patient is taking long-term APA therapy for either primary (presence of risk factors without a previous cardiovascular event) or secondary (previous myocardial infarction or stroke) cardiovascular prevention, a cardiovascular event may occur even when APA therapy is interrupted for only a few days. More specifically, the majority (60-70\%) of serious cardiovascular events develop within 10 days of APA interruption $[47,48]$. Consequently, APA suspension before endoscopy could be extremely dangerous in some patients, the consequences (infarction, stroke) being potentially life-threatening or leading to irreversible disability [46]. Therefore, the need for more appropriate management of APA therapy in patients who undergo endoscopy should be recognized.

\section{Antiplatelet therapy and urgent endoscopy}

APA therapy is one of the main causes of upper GI bleeding, as these drugs are capable of causing erosive/ulcerative lesions on both gastric and duodenal mucosa, as well as triggering a bleeding episode in a pre-existent lesion $[31,49]$. The role of APAs in causing colonic bleeding, mainly in diverticula, has been pointed out more recently [33]. Similarly, small bowel bleeding caused by APAs, mainly erosive enteropathy, is increasingly recognized [32].

The management of APAs in a patient who undergoes urgent endoscopy for bleeding essentially consists of the decision about when to suspend and restart antithrombotic therapy. This principally depends on whether the APA therapy is being administered for either primary or secondary cardiovascular prevention. Indeed, it has been calculated that the risk for ischemic events (myocardial infarction and stroke) without therapy is nearly 10 -fold higher in secondary than in primary prevention $(3.11 \%$ vs. $0.34 \%$ yearly) [46]. The current ESGE guideline on non-variceal upper GI bleeding recommends stopping low-dose aspirin in those patients on primary prevention and consulting the cardiologist for an evaluation of the risk/benefit of re-starting therapy when highrisk endoscopic stigmata are detected [10]. In contrast, patients on secondary prevention should resume APA therapy on day 3 following index endoscopy, preceded by a second-look endoscopy at the discretion of the endoscopist, based on lesion characteristics and/or confidence in effective hemostasis [10]. In patients receiving ongoing dual APA therapy, aspirin should be continued, whilst clopidogrel resumption/continuation needs to be decided on a case-by-case basis with immediate cardiology consultation [10]. In those patients with low-risk endoscopic stigmata (Forrest IIc and III), either single or dual APA therapy for secondary prevention should be continued, whereas the continuation of therapy for primary prevention needs to be reconsidered with the cardiologist. Switching from monotherapy with clopidogrel to aspirin should be also considered [10]. Notably, there is evidence that neither aspirin nor clopidogrel as ongoing therapy affects ulcer healing induced by proton pump inhibitors [50].
According to the recent American College of Gastroenterology (ACG) guideline for colonic bleeding, APAs should be discontinued when administered for primary, but not for secondary cardiovascular prevention [7]. Similarly, the ACG guideline on small-bowel bleeding management suggests APA discontinuation in all patients when possible, though with only a very low level of evidence [51]. However, APA therapy has been found to be an independent risk factor (HR 5.5, 95\% CI 1.8-16.8) for relapsing angiodysplasia bleeding through the GI tract [52].

\section{Anti-platelet therapy and elective endoscopy}

The management of APAs for elective endoscopy is based on a balanced evaluation of both procedure-related bleeding risk and patient-related thrombotic risk [47,53]. Endoscopic procedures are classified as high- and low-risk for bleeding, and the patients at high- and low-risk for thrombotic events, as reported in Fig. 1. Both the British Society of Gastroenterology (BSG)-ESGE and the ASGE guidelines recommend continuing APA therapy in patients undergoing elective endoscopy procedures associated with a low bleeding risk $[8,11]$. More specifically, the BSG-ESGE guideline recommends continuing aspirin for all endoscopic procedures with the exception of ESD, large colonic EMR ( $>2 \mathrm{~cm}$ ), upper GI EMR, and ampullectomy, for which the risk of thrombosis versus hemorrhage should be individually balanced [8]. Although such an approach is generally safe, it should be noted that a bleeding episode requiring endoscopic treatment following multiple gastroduodenal biopsies occurred in $2.2 \%$ of 45 healthy volunteers receiving aspirin or clopidogrel monotherapy [54]. In a prospective study, a bleeding episode requiring endoscopic treatment was observed in $2.2 \%$ of 1015 patients who underwent cold polypectomy for the removal of $<10 \mathrm{~mm}$ colonic polyps [56]. Notably, in this study APA monotherapy (aspirin or ticlopidine) was associated with a 4 -fold (95\% CI 1.5-10.6) increased risk of bleeding [55]. A recent systematic review found that the risk of a delayed, but not immediate, post-polypectomy hemorrhage was significantly increased in patients receiving APAs [56]: aspirin (odds ratio [OR] 1.7, 95\% CI 1.0-2.4), clopidogrel (OR 9.7, 95\% CI 3.1-30.8) and aspirin plus clopidogrel (OR 3.4, 95\% CI 1.3-8.8).

In patients receiving dual APA therapy, only the clopidogrel should be stopped 5 days before a high-risk endoscopic procedure when the thrombotic risk is low, whilst a cardiologist consultation is needed when the patient is at high thrombotic risk $[8,11]$. When the thrombotic risk following clopidogrel interruption is distinctly high (e.g., within 30 days after coronary stent placement) the operative procedure should be postponed [11]. Clopidogrel should be resumed after the procedure once hemostasis has been achieved during endoscopy [11].

Current guidelines suggest that endoscopic biopsies could also be performed in those patients on dual APA therapy $[8,11]$. However, data demonstrating the safety of performing gastroduodenal or colonic biopsies in patients receiving multiple antithrombotic agents are drawn from only a single 
study with 30 patients [57]. Notably, in this study only a mean of 1.68 biopsies were taken using thin-type biopsy forceps of 2-mm diameter. Although no delayed bleeding was observed, as many as 7-9 min were needed in $4(13.3 \%)$ cases until hemostasis occurred. Therefore, we should sound a note of caution, at least when multiple gastroduodenal or colonic biopsies are required, such as during upper endoscopy for anemia diagnostic workup (at least 4 duodenal and 5 gastric biopsies) or colonoscopy for diarrhea to exclude microscopic colitis. Indeed, the hemorrhagic risk increases with the number of biopsies [43].

\section{Concluding remarks}

APAs are very useful drugs that are widely used for both primary and secondary cardiovascular prevention. The undesired reverse side of the coin is that they are associated with an increased risk of GI hemorrhage, by causing erosive/ ulcerative lesions on the mucosa or inducing bleeding in an already existing lesion (angiodysplasia, ulcer, polyps, etc.). The probability of bleeding is particularly high in elderly subjects [58]. Moreover, the intrinsic action of APA, namely an irreversibleblock of platelet function, may cause post-endoscopy bleeding, particularly following an operative procedure. Unfortunately, APA therapy discontinuation, even for a short period ( $<10$ days), is associated with a significant increase in cardiovascular risk, especially in those patients taking APAs for secondary prevention [46]. Therefore, the endoscopist is required to balance the hemorrhagic and the cardiovascular risk for the individual patient [53]. On the whole, the clinical consequences of GI bleeding following an endoscopic procedure are less severe than those of a cardiovascular event. There is some evidence to suggest that endoscopists tend to overlook the thrombotic risk for patients when APA therapy is stopped. A recent survey showed that as many as $26 \%$ of 239 US endoscopists stated they would advise discontinuation of all APAs before performing any endoscopic procedure, including low-risk procedures, despite the recommendations of previous ASGE guidelines [59]. Even when the endoscopist possesses the expertise to judge the endoscopic bleeding risk on the one hand, on the other he/she may be not familiar with the criteria for evaluating the cardiovascular risk. Therefore, collaboration with the cardiologist is crucial, at least for the management of some patients. Fortunately, updated international guidelines are now available to aid the decision-making process in such an intricate field $[8,11]$.

\section{References}

1. Holbrook A, Schulman S, Witt DM, et al; American College of Chest Physicians. Evidence-based management of anticoagulant therapy: antithrombotic therapy and prevention of thrombosis, 9th ed: American College of Chest Physicians Evidence-Based Clinical Practice Guidelines. Chest 2012;141(2 Suppl):e152S-e184S.

2. Chugh SS, Havmoeller R, Narayanan K, et al. Worldwide epidemiology of atrial fibrillation: a Global Burden of Disease 2010
Study. Circulation 2014;129:837-847.

3. Douketis JD, Spyropoulos AC, Spencer FA, et al; American College of Chest Physicians. Perioperative management of antithrombotic therapy: antithrombotic therapy and prevention of thrombosis, 9th ed: American College of Chest Physicians Evidence-Based Clinical Practice Guidelines. Chest 2012;141 (2 Suppl):e326S-e350S.

4. Guerrouij M, Uppal CS, Alklabi A, Douketis JD. The clinical impact of bleeding during oral anticoagulant therapy: assessment of morbidity, mortality and post-bleed anticoagulant management. J Thromb Thrombolysis 2011;31:419-423.

5. Ansell J, Hirsh J, Hylek E, Jacobson A, Crowther M, Palareti G; American College of Chest Physicians. Pharmacology and management of the vitamin $\mathrm{K}$ antagonists: American College of Chest Physicians Evidence-Based Clinical Practice Guidelines (8th Edition). Chest 2008;133:160S-198S.

6. Marmo R, Koch M, Cipolletta L, et al; Italian registry on upper gastrointestinal bleeding (Progetto Nazionale Emorragie Digestive-PNED 2). Predicting mortality in non-variceal upper gastrointestinal bleeders: validation of the Italian PNED score and prospective comparison with the Rockall score. Am J Gastroenterol 2010;105:1284-1291.

7. Strate LL, Gralnek IM. ACG clinical guideline: management of patients with acute lower gastrointestinal bleeding. Am J Gastroenterol 2016;111:459-474.

8. Veitch AM, Vanbiervliet G, Gershlick AH, et al. Endoscopy in patients on antiplatelet or anticoagulant therapy, including direct oral anticoagulants: British Society of Gastroenterology (BSG) and European Society of Gastrointestinal Endoscopy (ESGE) guidelines. Endoscopy 2016;48:385-402.

9. Veitch AM, Vanbiervliet G, Gershlick AH, et al. Endoscopy in patients on antiplatelet or anticoagulant therapy, including direct oral anticoagulants: British Society of Gastroenterology (BSG) and European Society of Gastrointestinal Endoscopy (ESGE) guidelines. Gut 2016;65:374-389.

10. Gralnek IM, Dumonceau JM, Kuipers EJ, et al. Diagnosis and management of nonvariceal upper gastrointestinal hemorrhage: European Society of Gastrointestinal Endoscopy (ESGE) Guideline. Endoscopy 2015;47:a1-a46.

11. Acosta RD, Abraham NS, Chandrasekhara V, et al; ASGE Standards of Practice Committee. The management of antithrombotic agents for patients undergoing GI endoscopy. Gastrointest Endosc 2016;83:3-16.

12. Radaelli F, Paggi S, Terruzzi V, et al. Management of warfarinassociated coagulopathy in patients with acute gastrointestinal bleeding: a cross-sectional physician survey of current practice. Dig Liver Dis 2011;43:444-447.

13. Choudari CP, Rajgopal C, Palmer KR. Acute gastrointestinal haemorrhage in anticoagulated patients: diagnoses and response to endoscopic treatment. Gut 1994;35:464-466.

14. Wolf AT, Wasan SK, Saltzman JR. Impact of anticoagulation on rebleeding following endoscopic therapy for nonvariceal upper gastrointestinal hemorrhage. Am J Gastroenterol 2007;102:290-296.

15. Radaelli F, Dentali F, Repici A, et al. Management of anticoagulation in patients with acute gastrointestinal bleeding. Dig Liver Dis 2015;47:621-627.

16. Cuker A, Siegal DM, Crowther MA, Garcia DA. Laboratory measurement of the anticoagulant activity of the non-vitamin $\mathrm{K}$ oral anticoagulants. J Am Coll Cardiol 2014;64:1128-1139.

17. Pollack CV Jr, Reilly PA, Eikelboom J, et al. Idarucizumab for dabigatran reversal. N Engl J Med 2015;373:511-520.

18. Chai-Adisaksopha C, Hillis C, Monreal M, Witt DM, Crowther M. Thromboembolic events, recurrent bleeding and mortality after resuming anticoagulant following gastrointestinal bleeding. A meta-analysis. Thromb Haemost 2015;114:819-825.

19. Qureshi W, Mittal C, Patsias I, et al. Restarting anticoagulation and outcomes after major gastrointestinal bleeding in atrial fibrillation. 
Am J Cardiol 2014;113:662-668.

20. Douketis JD, Spyropoulos AC, Kaatz S, et al; BRIDGE Investigators. Perioperative bridging anticoagulation in patients with atrial fibrillation. N Engl J Med 2015;373:823-833.

21. http://www.clinicaltrials.gov/ct2/show/NCT00432796. [Accessed on September 26, 2016].

22. Friedland S, Sedehi D, Soetikno R. Colonoscopic polypectomy in anticoagulated patients. World J Gastroenterol 2009;15:1973-1976.

23. Horiuchi A, Nakayama Y, Kajiyama M, Tanaka N, Sano K, Graham DY. Removal of small colorectal polyps in anticoagulated patients: a prospective randomized comparison of cold snare and conventional polypectomy. Gastrointest Endosc 2014;79:417-423.

24. Spyropoulos AC, Turpie AG, Dunn AS, et al; REGIMEN Investigators. Perioperative bridging therapy with unfractionated heparin or low-molecular-weight heparin in patients with mechanical prosthetic heart valves on long-term oral anticoagulants (from the REGIMEN Registry). Am J Cardiol 2008;102:883-889.

25. Desai J, Kolb JM, Weitz JI, Aisenberg J. Gastrointestinal bleeding with the new oral anticoagulants-defining the issues and the management strategies. Thromb Haemost 2013;110:205-212.

26. Beyer-Westendorf J, Förster K, Pannach S, et al. Rates, management, and outcome of rivaroxaban bleeding in daily care: results from the Dresden NOAC registry. Blood 2014;124:955-962.

27. Healey JS, Eikelboom J, Douketis J, et al; RE-LY Investigators. Periprocedural bleeding and thromboembolic events with dabigatran compared with warfarin: results from the Randomized Evaluation of Long-Term Anticoagulation Therapy (RE-LY) randomized trial. Circulation 2012;126:343-348.

28. US Preventive Services Task Force. Aspirin for the prevention of cardiovascular disease: U.S. Preventive Services Task Force recommendation statement. Ann Intern Med 2009;150:396-404.

29. Morini S, Zullo A, Oliveti D, et al. A very high rate of inappropriate use of gastroprotection for nonsteroidal anti-inflammatory drug therapy in primary care: a cross-sectional study. J Clin Gastroenterol 2011;45:780-784.

30. Weisman SM, Graham DY. Evaluation of the benefits and risks of low-dose aspirin in the secondary prevention of cardiovascular and cerebrovascular events. Arch Intern Med 2002;162:2197-2202.

31. Lanas, Carrera-Lasfuentes P, Arguedas Y, et al. Risk of upper and lower gastrointestinal bleeding in patients taking nonsteroidal anti-inflammatory drugs, antiplatelet agents, or anticoagulants. Clin Gastroenterol Hepatol 2015;13:906-912.e2.

32. Endo H, Sakai E, Kato T, et al. Small bowel injury in low-dose aspirin users. J Gastroenterol 2015;50:378-386.

33. Yuhara H, Corley DA, Nakahara F, et al. Aspirin and non-aspirin NSAIDs increase risk of colonic diverticular bleeding: a systematic review and meta-analysis. J Gastroenterol 2014;49:992-1000.

34. Loperfido S, Baldo V, Piovesana E, et al. Changing trends in acute upper-GI bleeding: a population-based study. Gastrointest Endosc 2009;70:212-224.

35. Sbrozzi-Vanni A, Zullo A, Di Giulio E, et al. Low prevalence of idiopathic peptic ulcer disease: an Italian endoscopic survey. Dig Liver Dis 2010;42:773-776.

36. Lau JY, Sung J, Hill C, Henderson C, Howden CW, Metz DC. Systematic review of the epidemiology of complicated peptic ulcer disease: incidence, recurrence, risk factors and mortality. Digestion 2011;84:102-113.

37. Hallas J, Dall M, Andries A, et al. Use of single and combined antithrombotic therapy and risk of serious upper gastrointestinal bleeding: population based case-control study. BMJ 2006;333:726.

38. Lanas A, Gargallo CJ. Management of low-dose aspirin and clopidogrel in clinical practice: a gastrointestinal perspective. J Gastroenterol 2015;50:626-637.

39. Gouya G, Arrich J, Wolzt M, et al. Antiplatelet treatment for prevention of cerebrovascular events in patients with vascular diseases: a systematic review and meta-analysis. Stroke
2014;45:492-503.

40. Yamashita K, Arimura Y, Fukuda K, et al. Major bleeding after endoscopic biopsy in relation to use of antithrombotics. Endoscopy 2014;46:538.

41. Sheasgreen C, Leontiadis GI. Recent advances on the management of patients with non-variceal upper gastrointestinal bleeding. Ann Gastroenterol 2013;26:191-197.

42. Sarkis F, Abu Daya H, Sharara A, Soweid A, Barada K. Delayed overt gastrointestinal bleeding after cold endoscopic biopsy. Endoscopy 2013;45:75.

43. Domellöf L, Enander LK, Nilsson F. Bleeding as a complication to endoscopic biopsies from the gastric remnant after ulcer surgery. Scand J Gastroenterol 1983;18:951-954.

44. Hori M, Ikeda Y, Ishimaru S, et al. Guidelines for management of anticoagulant and antiplatelet therapy in cardiovascular disease (JCS 2009). 2009, published only online. http://wwwj-circorjp/ guideline/pdf/JCS_2009_hori_dpdf.

45. Miller AM, McGill D, Bassett ML. Anticoagulant therapy, antiplatelet agents and gastrointestinal endoscopy. J Gastroenterol Hepatol 1999;14:109-113.

46. Hassan C, Zullo A. Anti-platelet therapy and endoscopic procedures: eyes wide shut? Endosc Int Open 2015;3:E179-E180.

47. Biondi-Zoccai GG, Lotrionte M, Agostoni P, et al. A systematic review and meta-analysis on the hazards of discontinuing or not adhering to aspirin among 50,279 patients at risk for coronary artery disease. Eur Heart J 2006;27:2667-2674.

48. Sung JJ, Lau JY, Ching JY, et al. Continuation of low-dose aspirin therapy in peptic ulcer bleeding: a randomized trial. Ann Intern Med 2010;152:1-9.

49. Iwamoto J, Saito Y, Honda A, Matsuzaki Y. Clinical features of gastroduodenal injury associated with long-term low-dose aspirin therapy. World J Gastroenterol 2013;19:1673-1682.

50. Liu CP, Chen WC, Lai KH, et al; Formosa Acid-Related Disease (FARD) Study Group. Esomeprazole alone compared with esomeprazole plus aspirin for the treatment of aspirin-related peptic ulcers. Am J Gastroenterol 2012;107:1022-1029.

51. Gerson LB, Fidler JL, Cave DR, Leighton JA. ACG Clinical Guideline: diagnosis and management of small bowel bleeding. Am J Gastroenterol 2015;110:1265-1287.

52. Nardone G, Compare D, Scarpignato C, Rocco A. Long acting release-octreotide as "rescue" therapy to control angiodysplasia bleeding: A retrospective study of 98 cases. Dig Liver Dis 2014;46:688-694.

53. Belletrutti PJ, Heitman SJ. Management of anticoagulants and antiplatelet agents in elective endoscopy: weighing the risks and benefits. Can J Gastroenterol 2007;21:553-555.

54. Whitson MJ, Dikman AE, von Althann C, et al. Is gastroduodenal biopsy safe in patients receiving aspirin and clopidogrel?: a prospective, randomized study involving 630 biopsies. J Clin Gastroenterol 2011;45:228-233.

55. Repici A, Hassan C, Vitetta E, et al. Safety of cold polypectomy for $<10 \mathrm{~mm}$ polyps at colonoscopy: a prospective multicenter study. Endoscopy 2012;44:27-31.

56. Shalman D, Gerson LB. Systematic review with meta-analysis: the risk of gastrointestinal haemorrhage post-polypectomy in patients receiving anti-platelet, anti-coagulant and/or thienopyridine medications. Aliment Pharmacol Ther 2015;42:949-956.

57. Ono S, Fujishiro M, Kodashima S, et al. Evaluation of safety of endoscopic biopsy without cessation of antithrombotic agents in Japan. J Gastroenterol 2012;47:770-774.

58. Zullo A, Hassan C, Campo SM, Morini S. Bleeding peptic ulcer in the elderly: risk factors and prevention strategies. Drugs Aging 2007;24:815-828.

59. Kanakadandi V, Parasa S, Sihn P, et al. Patterns of antiplatelet agent use in the US. Endosc Int Open 2015;3:E173-E178. 